\title{
Treinamento de força como fator de proteção contra lesões: percepção de praticantes
}

\section{de musculação}

\author{
Strength training as a protective factor against injuries: perception of bodybuilders \\ El entrenamiento de fuerza como factor protector de lesiones: percepción de los culturistas
}

Recebido: 06/02/2022 | Revisado: 16/02/2022 | Aceito: 17/02/2022 | Publicado: 26/02/2022

Gabrielle da Silva Jordão

ORCID: https://orcid.org/0000-0002-3811-1246 Centro Universitário da Grande Dourados, Brasil Centro de Estudos e Pesquisas em Atividade Física e Saúde, Brasil Studio SER Personal e Reabilitação, Brasil E-mail: s.j.gabrielle@gmail.com

Joel Saraiva Ferreira

ORCID: https://orcid.org/0000-0003-4437-3100 Universidade Federal de Mato Grosso do Sul, Brasil Centro de Estudos e Pesquisas em Atividade Física e Saúde, Brasil E-mail: falecomjoel@hotmail.com

Fabiano Rodrigues Pissurno

ORCID: https://orcid.org/0000-0002-7290-8208 Centro Universitário da Grande Dourados, Brasil

E-mail: fabiano.pissurno@unigran.br

Raphael de Souza Cosmo

ORCID: https://orcid.org/0000-0001-7095-1782

Universidade Federal de Mato Grosso do Sul, Brasil

Centro Universitário da Grande Dourados, Brasil

E-mail: rafa-mig@ @otmail.com

Luis Eduardo Moraes Sinésio

ORCID: https://orcid.org/0000-0002-3839-1049 Instituto Federal de Educação, Ciência e Tecnologia de Mato Grosso do Sul, Brasil Centro Universitário da Grande Dourados, Brasil E-mail: luis.sinesio@unigran.br

Gildiney Penaves de Alencar

ORCID: https://orcid.org/0000-0002-5177-495X Universidade Federal de Mato Grosso do Sul, Brasil Centro de Estudos e Pesquisas em Atividade Física e Saúde, Brasil Centro Universitário da Grande Dourados, Brasil E-mail: gildiney.gpa@gmail.com

\begin{abstract}
Resumo
O objetivo deste estudo foi investigar a percepção de praticantes de musculação sobre o treinamento de força como fator de proteção contra lesões. Trata-se de um estudo observacional, de corte transversal, com abordagem quantitativa dos dados. A amostra, não estratificada por conveniência, foi composta por indivíduos adultos, de ambos os sexos, praticantes de treinamento de força em um studio localizado na cidade de Campo Grande/MS. A coleta de dados ocorreu no período de setembro a outubro de 2021, por meio de formulário online, distribuído aos participantes do estudo por meio de aplicativo de mensagem. Foram coletados dados sociodemográficos e a percepção dos indivíduos quanto à capacidade protetiva do treinamento de força contra lesões. Participaram do estudo 80 pessoas,

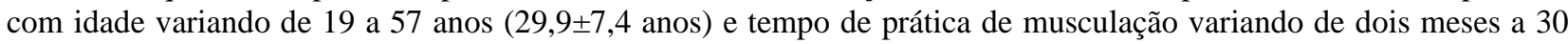
anos $(5,7 \pm 5,9$ anos). Houve maior prevalência de indivíduos do sexo feminino $(62,5 \%)$, na faixa etária de 18 a 40 anos $(90,0 \%)$, solteiros $(66,3 \%)$, pertencentes às classes C, D e E (90,0\%), praticantes de musculação há mais de 1 ano $(71,3 \%)$, numa frequência semanal de 3 a 5 dias $(78,7 \%)$, com duração das sessões de até 1 hora $(87,5 \%)$, em busca de saúde $(85,0 \%)$. Quase metade dos participantes $(45,0 \%)$ relatou a presença de lesões, sendo os joelhos a região mais afetada $(23,8 \%)$, seguida da coluna lombar $(11,3 \%)$, ombros $(11,3 \%)$ e tornozelo/pés $(10,0 \%)$. A maioria acredita que o treinamento de força contribui para prevenir ou melhorar as lesões $(98,8 \%)$, devido ao fortalecimento muscular, articular e melhora do condicionamento físico (66,3\%) e ao acompanhamento supervisionado e individualizado $(25,0 \%)$. O grupo avaliado entende que o treinamento de força, quando realizado sob orientação profissional personalizada, atua na proteção contra lesões, pois os exercícios físicos melhoram a aptidão física muscular e osteoarticular.
\end{abstract}

Palavras-chave: Treinamento de força; Lesões; Prevenção; Tratamento. 


\begin{abstract}
The objective of this study was to investigate the perception of bodybuilders about strength training as a protective factor against injuries. This is an observational, cross-sectional study with a quantitative approach to the data. The sample, not stratified by convenience, was composed of adult individuals, of both sexes, who practiced strength training in a studio located in the city of Campo Grande/MS. Data collection took place from September to October 2021, through an online form, distributed to study participants through a message application. Sociodemographic data and the perception of individuals regarding the protective capacity of strength training against injuries were collected. Eighty people participated in the study, with ages ranging from 19 to 57 years $(29.9 \pm 7.4$ years $)$ and bodybuilding practice ranging from two months to 30 years $(5.7 \pm 5.9$ years). There was a higher prevalence of female individuals (62.5\%), aged between 18 and 40 years (90.0\%), single (66.3\%), belonging to classes C, D and E (90.0\%), bodybuilders for more than 1 year $(71.3 \%)$, with a weekly frequency of 3 to 5 days $(78.7 \%)$, with sessions lasting up to 1 hour $(87.5 \%)$, in search of health $(85.0 \%)$. Almost half of the participants $(45.0 \%)$ reported the presence of injuries, with the knees being the most affected region (23.8\%), followed by the lumbar spine (11.3\%), shoulders $(11.3 \%)$ and ankle/feet $(10.0 \%)$. Most believe that strength training contributes to preventing or improving injuries (98.8\%), due to muscle and joint strengthening and improvement in physical conditioning $(66.3 \%)$ and supervised and individualized monitoring $(25.0 \%)$. The evaluated group understands that strength training, when performed under personalized professional guidance, acts to protect against injuries, as physical exercises improve muscular and osteoarticular physical fitness.
\end{abstract}

Keywords: Strength training; Injuries; Prevention; Treatment.

\title{
Resumen
}

El objetivo de este estudio fue investigar la percepción de los fisicoculturistas sobre el entrenamiento de fuerza como factor protector contra lesiones. Se trata de un estudio observacional, transversal, con abordaje cuantitativo de los datos. La muestra, no estratificada por conveniencia, fue compuesta por individuos adultos, de ambos sexos, que practicaban entrenamiento de fuerza en un estudio ubicado en la ciudad de Campo Grande/MS. La recolección de datos ocurrió de septiembre a octubre de 2021, a través de un formulario en línea, distribuido a los participantes del estudio a través de una aplicación de mensajes. Se recogieron datos sociodemográficos y la percepción de los individuos sobre la capacidad protectora del entrenamiento de fuerza frente a lesiones. Ochenta personas participaron en el estudio, con edades que oscilan entre 19 y 57 años $(29,9 \pm 7,4$ años) y práctica de musculación entre dos meses y

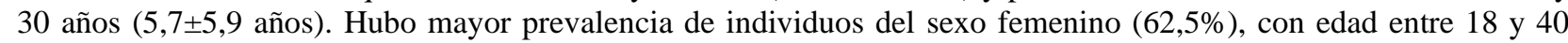
años $(90,0 \%)$, solteros $(66,3 \%)$, pertenecientes a las clases C, D y E (90,0 \%), fisicoculturistas hace más de 1 año $(71,3 \%)$, con una frecuencia semanal de 3 a 5 días $(78,7 \%)$, con sesiones de hasta 1 hora $(87,5 \%)$, en busca de la salud $(85,0 \%)$. Casi la mitad de los participantes $(45,0 \%)$ refirió la presencia de lesiones, siendo las rodillas la región más afectada $(23,8 \%)$, seguida de la columna lumbar $(11,3 \%)$, hombros $(11,3 \%)$ y tobillo/pie $(10,0 \%)$. La mayoría cree que el entrenamiento de fuerza contribuye a prevenir o mejorar las lesiones $(98,8 \%)$, debido al fortalecimiento muscular y articular y a la mejora de la condición física $(66,3 \%$ ) y al seguimiento supervisado e individualizado $(25,0 \%)$. El grupo evaluado entiende que el entrenamiento de fuerza, cuando se realiza bajo orientación profesional personalizada, actúa para proteger contra lesiones, ya que los ejercicios físicos mejoran la condición física muscular y osteoarticular.

Palabras clave: Entrenamiento de fuerza; Lesiones; Prevención; Tratamiento.

\section{Introdução}

O treinamento de força, treinamento com pesos, treinamento resistido ou musculação, corresponde ao uso de materiais e equipamentos como pesos ou halteres, que forneçam uma resistência ao indivíduo, com o objetivo de aperfeiçoar as capacidades físicas voltadas à saúde, ao desempenho atlético ou para fins estéticos (Baechle \& Groves, 2000).

A realização deste tipo de treinamento pode trazer diversos benefícios às pessoas, sejam saudáveis (Alencar et al., 2017; Paiva et al., 2019) ou com algum tipo de condição limitante (Pereira et al., 2020), dentre os quais pode-se citar os diferentes efeitos na saúde, como a redução do sobrepeso e obesidade, controle e prevenção do diabetes mellitus, redução dos fatores de risco para as doenças crônicas, melhora da mobilidade, entre outros (Murer, 2007).

Mesmo que seus benefícios sejam evidentes na atualidade, muitas vezes as pessoas se afastam da prática do treinamento de força devido à falsa narrativa de que ele provoca lesões e pode comprometer as estruturas corporais (Benedet et al., 2013). Entretanto, as lesões associadas à prática da musculação são mais frequentes em situações bem específicas e já relatadas na literatura (Murer, 2007), o que inclui o treinamento excessivo, falta de orientação profissional adequada, imperícia na execução das cargas de treino ou uso de equipamentos que não ofereçam segurança aos praticantes. 
Portanto, independentemente de qual for o objetivo do praticante, se o treinamento de força for realizado de forma segura, respeitando a individualidade biológica, controlando as variáveis que o envolvem e aplicando o princípio da sobrecarga progressiva, tal atividade produzirá benefícios, prevenindo e, muitas vezes, recuperando lesões já existentes (Souza et al., 2015; Alencar, 2018).

No entanto, grande parte das pesquisas envolvendo o treinamento de força e seus efeitos terapêuticos com lesões, é realizada com atletas (Souza et al., 2014; Rios et al., 2017; Cruz-Ferreira et al., 2015). Sendo assim, nota-se uma escassez de estudos voltados a compreender melhor tal relação com outros públicos. Além disso, torna-se importante obter informações sobre a percepção das pessoas quanto à temática, para que se tenha um melhor acompanhamento e prescrição desta modalidade de exercício para a população em geral (Souza et al., 2015), em especial para aquelas pessoas que realizam o treinamento de força de forma personalizada, como no caso dos studios de personal trainer, já que a procura por espaços dessa natureza vem crescendo devido à oferta dos serviços serem mais atrativas quanto à segurança e comodidade, em relação às academias tradicionais (Damasceno \& Damasceno, 2014).

Diante do exposto e da lacuna de conhecimento identificada, este estudo foi realizado com o objetivo de investigar a percepção de praticantes de musculação sobre o treinamento de força como fator de proteção contra lesões.

\section{Metodologia}

Trata-se de um estudo observacional de corte transversal (Gil, 2008) realizado com praticantes do treinamento de força não-atletas de um studio de musculação da cidade de Campo Grande, Mato Grosso do Sul.

Como critérios de inclusão, os participantes deveriam ser maiores de 18 anos, com ou sem histórico de lesões e estar praticando o treinamento de força no momento da entrevista.

A coleta de dados ocorreu entre os meses de setembro a outubro de 2021, com uso de um formulário online vinculado ao Google Formulários®, baseado no questionário aplicado por Baldissera et al. (2017) e Alencar et al. (2018), distribuído por aplicativo de mensagens, com questões voltadas à identificação das características sociodemográficas do público-alvo, bem como perguntas relacionadas à prática do treinamento de força e a percepção dos praticantes quanto ao seu efeito preventivo e terapêutico para as lesões.

O formulário online foi dividido em cinco seções, sendo elas: a) $\mathbf{1}^{\mathbf{a}}$ seção: Informações gerais da pesquisa e preenchimento do Termo de Consentimento Livre e Esclarecido (TCLE), com disponibilidade de uma via assinada pela pesquisadora principal ao participante; b) $\mathbf{2}^{\mathbf{a}}$ seção: Caracterização sociodemográfica (idade, sexo, estado civil e renda familiar per capita); c) $3^{\text {a }}$ seção: Características da prática do treinamento de força (tempo de prática, frequência, tempo das sessões, motivos que levam à prática, nível de motivação e satisfação); d) 4a seção: Percepção dos praticantes sobre o treinamento de força e lesões (se possui lesões, região corporal da lesão, acredita que o treinamento de força contribui e os benefícios proporcionados).

A estatística descritiva foi aplicada para a caracterização da amostra e as respostas obtidas pelo formulário, considerando as médias, apresentadas por meio de distribuição percentual e absoluta. Todos os dados foram tabulados com o uso do programa Microsoft Excel® e analisados por meio dos programas EPI-INFO ${ }^{\text {TM }}$ versão 7 (Centers Diseases Control and Prevention, Atlanta/Geórgia/EUA) e Bio Estat 5.3 (Sociedade Mamirauá, Belém/Pará/Brasil).

Este estudo integra a pesquisa "Observatório de Atividades Físicas e Esportivas" do Centro de Estudos e Pesquisas em Atividade Física e Saúde (CEPAFS) da Universidade Federal de Mato Grosso do Sul (UFMS) e faz parte da linha de pesquisa "Epidemiologia da atividade física e do comportamento sedentário", aprovada pelo Comitê de Ética em Pesquisa (CEP) em Seres Humanos da Universidade Federal de Mato Grosso do Sul sob o protocolo número 3.416.611 e obedeceu aos preceitos éticos determinados pela Resolução 466/12 do Conselho Nacional de Saúde (Brasil, 2012). 
Somente após esclarecimentos e garantia do sigilo dos dados, entendimento dos procedimentos da pesquisa e consentimento em participar da pesquisa, os participantes efetivaram sua colaboração voluntária com a pesquisa através da marcação eletrônica do Termo de Consentimento Livre e Esclarecido, sendo também disponibilizada uma via digital assinada pela pesquisadora principal no formulário online.

\section{Resultados}

Participaram da pesquisa 84 indivíduos, sendo excluídos os dados de quatro sujeitos (n=4) que não cumpriram os critérios de inclusão do estudo ou enviaram formulários incompletos.

A amostra final resultou em 80 praticantes de treinamento de força, com idades de 19 a 57 anos $(29,9 \pm 7,4$ anos). Dentre os participantes, foram mais prevalentes as pessoas do sexo feminino (62,5\%), na faixa etária de 18 a 40 anos - jovens adultos $(90,0 \%)$, solteiros $(66,3 \%)$, pertencentes às classes C, D e E - de acordo com a renda familiar per capita $(90,0 \%)$ (Tabela 1).

Tabela 1 - Variáveis sociodemográficas de praticantes de treinamento de força $(n=80)$.

\begin{tabular}{lcc}
\hline \multicolumn{1}{c}{ Variáveis } & $\mathbf{N}$ & $\%$ \\
\hline Total & 80 & 100 \\
Sexo & 50 & 62,5 \\
$\quad$ Feminino & 30 & 37,5 \\
$\quad$ Masculino & & \\
Faixa etária & 72 & 90,0 \\
$\quad$ 18 a 40 anos (jovem adulto) & 8 & 10,0 \\
Mais de 40 anos (meia-idade) & 53 & 66,3 \\
Estado civil & 22 & 27,5 \\
$\quad$ Solteiro & 5 & 6,2 \\
Casado ou União estável & 24 & \\
Divorciado & 24 & 30,0 \\
Renda familiar per capita & 24 & 30,0 \\
$\quad$ Até 2 SM (classe E) & 6 & 30,0 \\
De 2,1 a 4 SM (classe D) & 2 & 7,5 \\
De 4,1 a 10 SM (classe C) & & \\
De 10,1 a 20 SM (classe B) & & \\
Mais de 20 SM (classe A) & 2,5 \\
\hline
\end{tabular}

Nota: A faixa etária foi dividida de acordo as etapas de ciclo vital de Papalia, Olds e Feldman (2006); O salário mínimo (SM) utilizado para o cálculo da renda familiar per capita correspondente ao ano de 2021 e foi fixado em R $\$ 1.100,00$; A classificação das faixas salariais foi baseada no Critério Brasil (Associação Brasileira de Empresas de Pesquisa, 2019). Fonte: Autores (2022).

O tempo de prática do treinamento de força variou de dois meses a 30 anos, com média de 5,7 anos $( \pm 5,9)$, sendo que a maioria dos praticantes é classificada como avançados, pois realizam as sessões há mais de um ano (71,3\%), com uma frequência semanal de 3 a 5 dias $(78,7 \%)$ e tempo de duração de até 1 hora (87,5\%). Dentre os motivos, a maioria procura a musculação em busca de saúde $(85,0 \%)$, estética $(72,5 \%)$, qualidade de vida $(71,3 \%)$ e condicionamento físico $(70,0 \%)$. A maioria do grupo relatou satisfação com os resultados obtidos $(88,7 \%)$ e uma parte muito pequena dos avaliados não estava motivada com a prática realizada $(2,5 \%)$ (Tabela 2 ). 
Research, Society and Development, v. 11, n. 3, e36211326638, 2022

(CC BY 4.0) | ISSN 2525-3409 | DOI: http://dx.doi.org/10.33448/rsd-v11i3.26638

Tabela 2 - Distribuição da amostra com relação às características da prática do treinamento de força.

\begin{tabular}{|c|c|c|}
\hline Variáveis & $\mathbf{N}$ & $\%$ \\
\hline Total & 80 & 100 \\
\hline \multicolumn{3}{|l|}{ Tempo de prática } \\
\hline Iniciante (até 6 meses) & 11 & 13,7 \\
\hline Intermediário (de 6,1 meses a 1 ano) & 12 & 15,0 \\
\hline Avançado (mais de 1 ano) & 57 & 71,3 \\
\hline \multicolumn{3}{|l|}{ Frequência semanal } \\
\hline 1 a 2 dias & 4 & 5,0 \\
\hline 3 a 5 dias & 63 & 78,7 \\
\hline 6 a 7 dias & 13 & 16,3 \\
\hline \multicolumn{3}{|l|}{ Duração das sessões } \\
\hline Até 1 hora & 70 & 87,5 \\
\hline Mais de 1 hora & 10 & 12,5 \\
\hline \multicolumn{3}{|l|}{ Motivos que levam a praticar musculação } \\
\hline Saúde & 68 & 85,0 \\
\hline Estética & 58 & 72,5 \\
\hline Qualidade de vida & 57 & 71,3 \\
\hline Condicionamento físico & 56 & 70,0 \\
\hline Lazer & 25 & 31,3 \\
\hline Socialização & 13 & 16,3 \\
\hline Recomendação médica & 10 & 12,5 \\
\hline Outros & 8 & 10,0 \\
\hline \multicolumn{3}{|l|}{ Se sentem satisfeitos com os resultados } \\
\hline Sim & 71 & 88,7 \\
\hline Não & 9 & 11,3 \\
\hline \multicolumn{3}{|l|}{ Nível de motivação } \\
\hline Nada motivado & 2 & 2,5 \\
\hline Muito pouco motivado & 7 & 8,7 \\
\hline Motivado & 37 & 46,3 \\
\hline Muito motivado & 22 & 27,5 \\
\hline Completamente motivado & 12 & 15,0 \\
\hline
\end{tabular}

Nota: A classificação do tempo de prática foi realizada com base no estudo de Vieira e Fernandes (2005); Em relação aos motivos que levam a praticar musculação os participantes puderam marcar uma ou mais opções; A classificação do nível de motivação foi adaptada de Alencar, Marin, Lima e Teixeira (2018). Fonte: Autores (2022).

Houve predomínio de praticantes que não possuem lesões (55,0\%). Entretanto, 45\% dos participantes relataram a presença de lesões, sendo os joelhos a região mais afetada (23,8\%), seguida da coluna lombar $(11,3 \%)$, ombros (11,3\%) e tornozelo/pés (10,0\%) (Tabela 3). 
Tabela 3 - Distribuição da amostra com relação à presença/ausência de lesões e regiões corporais afetadas.

\begin{tabular}{lcc}
\hline \multicolumn{1}{c}{ Variáveis } & $\mathbf{N}$ & $\mathbf{\%}$ \\
\hline Total & 80 & 100 \\
Possui lesões & 36 & 45,0 \\
$\quad$ Sim & 44 & 55,0 \\
$\quad$ Não & & 23,8 \\
Região corporal afetada por lesões & 19 & 11,3 \\
$\quad$ Joelhos & 9 & 11,3 \\
Coluna lombar & 9 & 10,0 \\
Ombros & 8 & 5,0 \\
Tornozelo/pés & 4 & 3,8 \\
Cotovelos & 3 & 2,5 \\
Coluna cervical & 2 & 2,5 \\
Coluna torácica & 2 & 2,5 \\
Antebraços & 2 & 2,5 \\
Coxas & 2 & 1,3 \\
Pernas & 1 & \\
Punhos/mãos & 44 & 55,0 \\
Não se aplica & & \\
\hline
\end{tabular}

Nota: Os participantes que reportaram a presença de lesões puderam marcar uma ou mais opções quanto às regiões afetadas.

Fonte: Autores (2022).

Apesar da maioria dos participantes não relatarem lesões, grande parte do público avaliado (98,8\%) compreende que o treinamento de força realizado no studio de musculação contribui para prevenir ou melhorar as condições de lesões existentes. Dentre as principais justificativas para essa prevenção ou melhora das lesões, se destacam o fortalecimento muscular, articular e melhora do condicionamento físico (66,3\%), o acompanhamento supervisionado e individualizado ofertado (25,0\%), além da redução das dores $(15,0 \%)$ e execução da postura e técnicas adequadas durante as sessões $(10,0 \%)$ (Tabela 4).

Tabela 4 - Distribuição da amostra com relação à percepção dos praticantes sobre o treinamento de força e lesões.

\begin{tabular}{lcc}
\hline \multicolumn{1}{c}{ Variáveis } & $\mathbf{N}$ & $\mathbf{\%}$ \\
\hline Total & 80 & 100 \\
Acredita que o treinamento de força no studio contribui para & & \\
prevenir ou melhorar lesões & 79 & 98,8 \\
$\quad$ Sim & 1 & 1,2 \\
Não & 53 & 66,3 \\
Justificativa da contribuição na prevenção e melhora de lesões & 20 & 25,0 \\
$\quad$ Fortalecimento muscular, articular e condicionamento & 12 & 15,0 \\
Acompanhamento supervisionado e individualizado & 8 & 10,0 \\
Redução das dores & & \\
Postura e técnicas adequadas & & \\
\end{tabular}

Fonte: Autores (2022).

Quanto aos benefícios gerais do treinamento de força no dia a dia, a maioria dos participantes relatou que as sessões possibilitam uma melhora da disposição, do humor, redução do estresse e da ansiedade (65,0\%), além de aumentar a força, o condicionamento físico, reduzir as dores osteomusculares $(28,7 \%)$, promover o bem-estar e uma melhor qualidade de vida $(21,3 \%)$ e contribuir para a autoestima e a imagem corporal (20,0\%). Quando os avaliados foram questionados sobre a sensação obtida na ausência do treinamento de força, a maioria relatou que há uma elevação do cansaço físico, emocional, indisposição e frustração $(73,7 \%)$ (Tabela 5). 
Tabela 5 - Distribuição da amostra com relação aos benefícios gerais do treinamento de força e a opinião sobre a ausência de treinamento.

\begin{tabular}{lcc}
\hline \multicolumn{1}{c}{ Variáveis } & $\mathbf{N}$ & $\mathbf{\%}$ \\
\hline Total & 80 & 100 \\
Benefícios gerais & 52 & 65,0 \\
Melhora a disposição, o humor, reduz o estresse e a ansiedade & 23 & 28,7 \\
Aumenta a força, o condicionamento físico e reduz as dores osteomusculares & 17 & 21,3 \\
Promove o bem-estar e uma melhor qualidade de vida & 16 & 20,0 \\
Contribui para a autoestima e a imagem corporal & 9 & 11,3 \\
Favorece a postura e o sono & 7 & 8,7 \\
Eleva as condições de saúde e a imunidade & 59 & 73,7 \\
O que sente na ausência de treinamento? & 6 & 7,5 \\
Cansaço, indisposição e frustração & 4 & 5,0 \\
Aumento do estresse, do mal humor e da ansiedade & \\
Dores e dificuldade para dormir & & \\
\hline
\end{tabular}

Fonte: Autores (2022).

\section{Discussão}

De forma geral, foi observado que os praticantes acreditam que o treinamento de força auxilia na prevenção e melhora das lesões e produz efeitos positivos na disposição, na redução das dores e na melhora da qualidade de vida pelo fato do atendimento individualizado proporcionado.

Esses achados corroboram com Damasceno e Damasceno (2014), pois os studios de personal trainer têm se tornado um ambiente atrativo devido à segurança e a comodidade, em comparação com as academias tradicionais, além dos profissionais conseguirem direcionar uma atenção mais especial, o que gera melhores resultados aos indivíduos que treinam nesses ambientes.

Dentre os 80 praticantes do treinamento de força que participaram desse estudo, verificou-se um maior envolvimento das mulheres (62,5\%), na faixa etária de 18 a 40 anos, resultado observado também por Strub e Limberger (2015) ao avaliarem o perfil de usuários de musculação em duas regiões da cidade de Santa Cruz do Sul, Rio Grande do Sul. Entretanto, a maior participação do público jovem adulto do sexo feminino não parece ser exclusiva da musculação, já que em Campo Grande, Mato Grosso do Sul, houve uma maior presença desse público na modalidade de treinamento funcional (Alencar et al., 2018).

Quanto à aderência dos participantes solteiros (66,3\%), apesar de não ser uma regra, o envolvimento com tarefas domésticas e trabalhistas podem interferir na realização de atividade física, desta forma, esse fato pode explicar a maior adesão dessas pessoas por terem "tempo" para dedicar a esta prática (Liz \& Andrade, 2016).

Apesar de Rodrigues, Melo, Assis e Palma (2017) identificarem que as condições socioeconômicas influenciam na prática de atividade física, de modo que aqueles indivíduos com menor renda se envolvem mais em atividade físicas de deslocamento e/ou ocupacionais, enquanto o grupo de maior renda tem maior envolvimento em atividade físicas no lazer, a participação dos praticantes pertencentes às classes C, D e E foi maior neste estudo (90,0\%). Isso mostra que a procura por treinamento físico individualizado tem crescido nas classes menos favorecidas, principalmente aos que ganham até 4 salários mínimos, entretanto, ainda precisam ser pensadas estratégias para garantir a promoção de atividade física para os grupos de menor vulnerabilidade (Rodrigues et al., 2020).

Quando nos referimos ao tempo de prática, temos maior prevalência de praticantes avançados $(71,3 \%)$, que estão há mais de um ano na prática de musculação. Porém, não foi questionado se neste período ocorreram desistências e retornos à atividade, o que representa uma limitação. Dentre os motivos os quais levaram estes participantes à pratica, a saúde foi elencada com maior índice (85\%), seguida da estética (72,5\%), qualidade de vida (71,3\%) e condicionamento físico (70\%). O fato da saúde estar em primeiro lugar seria uma justificativa para a constância na modalidade, pois se mostra necessária no dia 
a dia do praticante, além da estética, qualidade de vida e condicionamento físico também serem objetivos comuns para quem procura esta modalidade de exercício (Strub \& Limberger, 2015).

Em relação a frequência semanal, a maior parte dispõe de 3 a 5 dias para realização da atividade física $(78,7 \%)$ e o tempo de 1 hora por sessão (87,5\%). Esse limiar de frequência e duração das sessões pode ter relação com a jornada de trabalho e com o tempo despendido com as obrigações familiares, permitindo maior disponibilidade de uso do tempo de lazer com a prática de atividades físicas (Liz \& Andrade, 2016). No mais, os participantes deste estudo cumprem com as recomendações da Organização Mundial da Saúde e do Guia de Atividade para a População Brasileira, já que adultos entre os 18 e 64 anos devem realizar o fortalecimento muscular por dois ou mais dias na semana aliadas às atividades aeróbicas por 150 a 300 minutos semanais ou 75 a 150 minutos com intensidade vigorosa (World Health Organization, 2020; Brasil, 2021).

Os participantes encontram-se motivados $(46,3 \%)$ e satisfeitos $(88,7 \%)$ com os resultados obtidos da prática. Tais resultados podem ser oriundos exatamente da particularidade do tipo de local onde os exercícios são praticados, que no caso é um studio com treinamento individualizado, implicando substancialmente na forma de atendimento, sendo de grande importância a motivação do profissional para com o aluno, para este permanecer ativo (Saboya \& Brandão, 2013).

Quase metade dos participantes apresentou algum tipo de lesão (45\%), sendo as mais frequentes, nos joelhos (23,8\%), na coluna lombar e ombros $(11,3 \%)$, tornozelos/pés (10\%), que podem ser causadas pelos mais variados motivos como a rotina deste praticante, sessões exaustivas de trabalho, movimentos repetitivos de determinadas articulações (Verthein \& Gomez, 2011), e devem ser levadas em conta na prescrição dos treinamentos.

Desta forma, a prática do treinamento de força é amplamente recomendada e reafirmada pelos participantes da pesquisa, pois ajuda no tratamento e prevenção de lesões (98,8\%), como também relata Liz e Andrade (2016), principalmente quando o treinamento é específico e o acompanhamento profissional é realizado.

Além disso, é notório que o exercício físico e o treinamento de força influenciam no bem-estar psicológico, na autoestima, na imagem corporal (Hausenblas \& Fallon, 2006), no controle do estresse (Segato et al., 2010), na redução da ansiedade, da depressão e na melhora do sono (Viais, 2015), o que também foi relatado no studio de musculação onde foi realizada a coleta de dados, já que os participantes notaram uma melhora da disposição, do humor, redução do estresse e da ansiedade $(65,0 \%)$, aumento da força, do condicionamento físico, redução das dores osteomusculares $(28,7 \%)$, promoção do bem-estar, qualidade de vida (21,3\%) e contribuição para a autoestima e a imagem corporal (20,0\%).

Ainda, para reforçar esses benefícios, na ausência da realização do treinamento de força os participantes relataram que há uma elevação do cansaço físico, emocional, indisposição e frustração (73,7\%), além de aumentar o estresse, o mal humor, a ansiedade (7,5\%), dores e dificuldades para dormir (5,0\%), elementos que exprimem a importância global da prática do treinamento de força de forma individualizada.

\section{Conclusão}

Os praticantes do treinamento de força percebem que este treinamento auxilia na prevenção e melhora das lesões, proporciona benefícios para a saúde física, mental e social, sendo de grande importância para vivência de suas atividades diárias.

O treinamento de força proporciona benefícios amplos para a prevenção e melhora de lesões corporais, com destaque para a percepção de melhora do fortalecimento muscular, articular e condicionamento físico, além da redução das dores devido ao acompanhamento supervisionado e individualizado ofertado, execução da postura e técnica adequada durante as sessões.

Por fim, sugere-se a realização de novos estudos que comparem a percepção dos praticantes de treinamento de força em studios de musculação e em academias convencionais, para que seja ampliado o escopo de conhecimentos sobre o treinamento com pesos e seus efeitos terapêuticos sobre as lesões. 


\section{Agradecimentos}

Os autores agradecem ao Centro Universitário da Grande Dourados (UNIGRAN EAD), ao Centro de Estudos e Pesquisas em Atividade Física e Saúde da Universidade Federal de Mato Grosso do Sul (CEPAFS/UFMS), ao studio de musculação e a todos os participantes voluntários da pesquisa.

\section{Referências}

Associação Brasileira de Empresas de Pesquisa. (2019). Critério de classificação econômica Brasil. Alterações na aplicação do Critério Brasil, válidas a partir de 01/09/2020. https://www.abep.org/criterio-brasil

Alencar, G. P., Gonçalves, J. L., Lima, L. E. M., \& Souza, A. F. (2017). Comparação das cargas de treinamento nos testes de repetições máximas e 1RM em indivíduos praticantes do treinamento de força. Revista Brasileira de Fisiologia do Exercício, 16 (3), 189-193. https://doi.org/10.33233/rbfe.v16i3.1142

Alencar, G. P. (2018). Relevância do treinamento de força e suas variáveis nos mais diversos objetivos. FIEP Bulletin, 88 (Especial), $341-344$. http://www.fiepbulletin.net/index.php/fiepbulletin/article/view/6010

Alencar, G. P., Marin, J. L. O., Lima, L. E. M., \& Teixeira, C. V. L. S. (2018). Perfil de praticantes de treinamento funcional de Campo Grande/MS. Revista Brasileira de Fisiologia do Exercício, 17 (2), 80-85. https://doi.org/10.33233/rbfe.v17i2.2465

Baechle, T. R., \& Groves, B. R. (2000). Treinamento de força: passos para o sucesso (2a ed.). Artmed.

Baldissera, L., Machado, D. L., Alves, L. G., Faleiro, D.; \& Zawadzki, P. (2017). Benefícios percebidos por praticantes de musculação para a saúde, estilo de vida e qualidade de vida. Unoesc \& Ciência - ACBS Joaçaba, 8 (2), 117-124. https://portalperiodicos.unoesc.edu.br/acbs/article/view/13055

Benedet, J., Freddi, J. C., Luciano, A. P., Almeida, F. S., Silva, G. L.; Hinnig, P. F., \& Adami, F. (2013). Resistance training for children and adolescents. ABCS Health Sciences, 38 (1), 40-46. https://doi.org/10.7322/abcshs.v38i1.7

Brasil. Ministério da Saúde. Conselho Nacional de Saúde. (2012). Resolução n. 466, de 12 de dezembro de 2012. Aprova diretrizes e normas regulamentadoras de pesquisas envolvendo seres humanos. Brasília, Ministério da Saúde. https://conselho.saude.gov.br/resolucoes/2012/Reso466.pdf

BRASIL. Ministério da Saúde. Secretaria de Atenção Primária à Saúde. Departamento de Promoção da Saúde. (2021). Guia de Atividade Física para a População Brasileira. Brasília: Ministério da Saúde. http://bvsms.saude.gov.br/bvs/publicacoes/guia_atividade_fisica_populacao_brasileira.pdf

Cruz-Ferreira, A., Marujo, A., Folgado, H., Gutierres Filho, P., \& Fernandes, J. (2015). Programas de exercício na prevenção de lesões em jogadores de futebol: uma revisão sistemática. Revista Brasileira de Medicina do Esporte, 21 (3), 236-241. http://dx.doi.org/10.1590/1517-86922015210302174

Damasceno, M. C., \& Damasceno, G. J. (2014). Fatores e características determinantes que praticantes de musculação buscam para contratar um personal trainer em um Studio da cidade de Caratinga, MG. Revista Lecturas, Educación Física y Deportes, 19 (195), 1-7. https://www.efdeportes.com/efd195/fatorespara-contratar-um-personal-trainer.htm

Gil, A. C. (2008). Métodos e técnicas de pesquisa social. (6a ed.). Atlas.

Hausenblas, H. A., \& Fallon, E. A. (2006). Exercise and body image: a meta-analysis. Psychology and Health, 21 (1), 33-47. https://doi.org/10.1080/14768320500105270

Liz, C. M., \& Andrade, A. (2016). Análise qualitativa dos motivos de adesão e desistência da musculação em academias. Revista Brasileira de Ciências do Esporte, 38 (3), 267-274. http://dx.doi.org/10.1016/j.rbce.2015.11.005

Murer, E. Epidemiologia da musculação. In: Roberto, V. (Org.). (2007). Saúde coletiva e atividade física: conceitos e aplicações dirigidos à graduação em Educação Física. Campinas: IPES Editorial. https://www.fef.unicamp.br/fef/sites/uploads/deafa/qvaf/saude_coletiva_completo.pdf

Paiva, M. R., Rodrigues, G. C. G., Pereira, T. T., Alencar, G. P., Lima, L. E. M., Krug, A. L. O., \& Souza, A. F. (2019). Respostas agudas da pressão arterial em exercícios básicos do treinamento de força. Revista Brasileira de Fisiologia do Exercício, 18 (1). https://doi.org/10.33233/rbfe.v18i1.2532

Papalia, D. E., Olds, S. W., \& Feldman, R. D. (2006). Desenvolvimento humano. (8a ed.). Artmed.

Pereira, M. G. L., Vincensi, I., Alencar, G. P., \& Lima, L. E. M. (2020). Efeitos do treinamento de força no consumo máximo de oxigênio em indivíduos cardiopatas sedentários. Revista Contribuciones a Las Ciencias Sociales, enero. https://www.eumed.net/rev/cccss/2020/01/individuos-cardiopatassedentarios.html

Rodrigues, P., Reis, E. C., Bianchi, L., \& Palma, A. (2020). Fatores associados à prática de atividades físicas durante a pandemia da COVID-19 no estado do Rio de Janeiro, Brasil. Revista Brasileira de Atividade Física e Saúde, 25 (e0124). https://doi.org/10.12820/rbafs.25e0124

Rodrigues, P. A. F., Melo, M. P., Assis, M. R., \& Palma, A. (2017). Condições socioeconômicas e prática de atividades físicas em adultos e idosos: uma revisão sistemática. Revista Brasileira de Atividade Física e Saúde, 22 (3), 217-232. https://doi.org/10.12820/rbafs.v.22n3p217-232

Rios, E. T., Rodrigues, F. C., Rocha, L. F., Salemi, V. M. C., \& Miranda, D. P. (2017). Influência do volume semanal e do treinamento resistido sobre a incidência de lesão em corredores de rua. Revista Brasileira de Prescrição e Fisiologia do Exercício, 11 (64), 104-109. http://www.rbpfex.com.br/index.php/rbpfex/article/view/1086

Saboya, P. J. T., \& Brandão, D. C. (2013). Análise dos aspectos motivacionais dos alunos praticantes de musculação. Revista Lecturas, Educación Física y Deportes, 18 (179), 1-4. https://www.efdeportes.com/efd179/aspectos-motivacionais-de-musculacao.htm 
Research, Society and Development, v. 11, n. 3, e36211326638, 2022

(CC BY 4.0) | ISSN 2525-3409 | DOI: http://dx.doi.org/10.33448/rsd-v11i3.26638

Segato, L., Brandt, R., Liz, C. M., Vasconcellos, D. I. C., \& Andrade, A. (2010). Estresse psicológico de velejadores de alto nível esportivo em competição. Motricidade, 6 (3), 53-62. http://www.redalyc.org/articulo.oa?id=273019708007

Souza, C. A. B., Santos, C., Aquino, F. A. O., Monteiro, I. O., Azevedo, M. V. G. T., Buongermino, A. R., \& Dourado, V. Z. (2014). Treinamento resistido como fator preventivo de lesões em corredores dos $10 \mathrm{~km}$ de Tribuna FM, Unilus. Revista Lecturas, Educación Física y Deportes, 19 (197), 1-7. https://www.efdeportes.com/efd197/preventivo-de-lesoes-em-corredores-dos-10-km.htm

Souza, G. L., Moreira, N. B., \& Campos, W. (2015). Ocorrência e características de lesões entre praticantes de musculação. Revista Saúde e Pesquisa, 8 (3), 469-477. https://periodicos.unicesumar.edu.br/index.php/saudpesq/article/view/4476/2695

Strub, A., \& Limberger, O. J. (2015). Perfil de usuários de musculação: um olhar sobre o comportamento no exercício físico de praticantes de academias de regiões opostas de Santa Cruz do Sul, RS, Brasil. Revista Lecturas, Educación Física y Deportes, 19 (202), 1-6. https://www.efdeportes.com/efd202/perfil-deusuarios-de-musculacao-de-academias.htm

Verthein, M. A. R., \& Gomez, C. M. (2011). Movimentos de Assepsia Social: a doença do trabalho fora de alcance. In: Gomez, C. M., Machado, J. M. H., Pena, P. G. L. Saúde do trabalhador na sociedade brasileira contemporânea. Rio de Janeiro: Editora FIOCRUZ. https://books.scielo.org/id/qq8zp/pdf/minayo9788575413654-14.pdf

Viais, A. S. (2015). Benefícios da prática da musculação na imagem corporal para mulheres. FACIDER Revista Científica, IX (9), 1-14. http://revista.seicesucol.edu.br/index.php/facider/article/view/141

Vieira, A. P., \& Souza, A. F. (2005). A ordem dos exercícios no treinamento de força, como fator modificador da intensidade. Anais do Congresso de Ciências do Desporto, Campinas, SP, Brasil, 1. https://www.fef.unicamp.br/fef/sites/uploads/congressos/ccd2005/temalivre/allexpradovieira.pdf

World Health Organization. (2020). Physical activity: Fact Sheet of 26 November 2020. World Health Organization. https://www.who.int/news-room/factsheets/detail/physical-activity 